ESAIM: PROCEEDINGS, December 2012, Vol. 38, p. 183-201

F. Coquel, M. Gutnic, P. Helluy, F. Lagoutière, C. Rohde, N. Seguin, Editors

\title{
OSAMOAL: OPTIMIZED SIMULATIONS BY ADAPTED MODELS USING ASYMPTOTIC LIMITS *
}

\author{
Anne-Céline Boulanger ${ }^{1}$, Clément Cancès $^{2}$, Hélène Mathis $^{3}$, Khaled Saleh $^{2}$ \\ AND Nicolas SEguin ${ }^{2}$
}

Dedicated to "La côte de bœuf", 35 Cours Honoré d'Estienne d'Orves, Marseille.

\begin{abstract}
We propose in this work to address the problem of model adaptation, dedicated to hyperbolic models with relaxation and to their parabolic limit. The goal is to replace a hyperbolic system of balance laws (the so-called fine model) by its parabolic limit (the so-called coarse model), in delimited parts of the computational domain. Our method is based on the construction of asymptotic preserving schemes and on interfacial coupling methods between hyperbolic and parabolic models. We study in parallel the cases of the Goldstein-Taylor model and of the $p$-system with friction.
\end{abstract}

Résumé. Nous proposons dans ce travail de traiter le problème d'adaptation de modèle, appliqué aux systèmes hyperboliques de relaxation et à leur limite parabolique. Le but est de remplacer dans des zones délimitées du domaine de calcul un système hyperbolique avec terme source (le modèle fin) par le modèle parabolique limite associé (le modèle grossier). Notre méthode repose sur des schémas préservant cette asymptotique et le couplage interfacial entre des modèles hyperbolique et parabolique. On étudie les cas du modèle de Goldstein-Taylor et du p-système avec friction avec leurs limites paraboliques respectives.

\section{Contents}

$\begin{array}{lr}\text { Introduction } & 184\end{array}$

1. Models and asymptotic limits 184

1.1. The Goldstein-Taylor model $r$

1.2. The $p$-system $r$

2. Asymptotic preserving schemes 185

2.1. The Goldstein-Taylor model $\quad 186$

\begin{tabular}{ll}
2.2. & The $p$-system \\
\hline
\end{tabular}

3. Interface coupling between the fine model and the coarse model 196

3.1. The Goldstein-Taylor model 196

\begin{tabular}{lr}
3.2. & The $p$-system \\
\hline
\end{tabular}

* This project has been supported by the LRC Manon (Modélisation et Approximation Numérique Orientées pour l'énergie Nucléaire - CEA/DM2S-LJLL).

${ }^{1}$ INRIA Paris-Rocquencourt, Équipe-projet BANG, Domaine de Voluceau, 78153, Le Chesnay, France

${ }^{2}$ UPMC Univ Paris 06 \& CNRS, UMR 7598, Laboratoire Jacques-Louis Lions, F-75005, Paris, France

${ }^{3}$ Université de Nantes, Laboratoire de mathématiques Jean Leray, 2 rue de la Houssinière, 44322 Nantes, France

(C) EDP Sciences, SMAI 2012 
4. Conclusion $r$

References

\section{INTRODUCTION}

In simulation of complex flows for industrial purposes, it is natural to replace an accurate model by a simpler one in the regions of the computational domain where the flow is supposed to be almost at rest for the sake of reducing the computational cost. Let us give an example. Assume that the flow under consideration is a twophase flow, composed by air and water. In the highly heterogeneous regions, a complex model which accounts for phenomena with very small time and space scales has to be used such as the Baer-Nunziato model [2] where each phase is described by its own density, velocity and pressure and global relaxation terms represent the effects of the differences of velocity and pressure. In some smoother regimes, drift-flux models can be used [18], where the pressures are equal and the relative velocity between the phases is a given function of the characteristic variable describing the flow (densities of each phases, mean velocity and mean pressure).

These regions where the simpler (or coarser) model can be used can be determined empirically or by the use of preliminary computations. In this work, we continue the program initiated in [21] and [5] about model adaptation. This techniques rely on automatically selecting the "good" model among a hierarchy of models, compatible by asymptotic arguments. The notion of "good" model means that we want to maximize the local use of coarse models without deteriorating the accuracy of the numerical results. Here, we focus on parabolic limits of hyperbolic models, while hyperbolic limits were studied in [21] and [5]. We restrict ourselves to standard systems and we do not investigate the dynamic part of the adaptation: we focus on the construction of numerical schemes which preserve the parabolic asymptotic limit and on the interfacial coupling between the hyperbolic system and its parabolic limit.

In the first section, we present the two models we are dealing with: the Goldstein-Taylor model and the $p$-system with friction. We also provide their asymptotic limit, which turns out to be a parabolic equation. In the second section, we derive and analyze numerical schemes which are able to reproduce the parabolic limits at the discrete level, namely asymptotic preserving schemes. Up to our knowledge, the results on the $p$-system are new. Note that we try to provide accurate stability estimates in order to fully understand the robustness of the CFL condition when $\varepsilon \rightarrow 0$. The next part is devoted to the coupling between the hyperbolic model and its parabolic limit through a fixed thin interface. It has to be done carefully because classical methods of coupling fails to give accurate results. In order to illustrate our developments, preliminary numerical illustrations are also provided.

\section{Models AND ASYMptotic Limits}

\subsection{The Goldstein-Taylor model}

Let us consider the following fine model, called the Telegraph equation, the Goldstein-Taylor system or the p-system with friction for a Chaplygin gas:

$$
\left\{\begin{array}{l}
\varepsilon \partial_{t} v+\partial_{x} u=0, \\
\varepsilon \partial_{t} u+a^{2} \partial_{x} v=\frac{-\sigma}{\varepsilon} u,
\end{array}\right.
$$

where $\sigma$ is a positive friction coefficient and $a$ the sound speed. 
An asymptotic analysis when $\varepsilon \rightarrow 0$, using the Chapman-Enskog expansion for instance, leads to a coarse model, which is nothing but the heat equation:

$$
\left\{\begin{array}{l}
\partial_{t} v-\frac{a^{2}}{\sigma} \partial_{x x} v=0 \\
u=0
\end{array}\right.
$$

\subsection{The $p$-system}

Let us now consider the $p$-system with a friction term

$$
\left\{\begin{array}{l}
\varepsilon \partial_{t} \tau-\partial_{x} u=0, \\
\varepsilon \partial_{t} u+\partial_{x} P(\tau)=\frac{-\sigma}{\varepsilon} u,
\end{array}\right.
$$

where $\tau$ is the specific volume, $u$ the velocity and $\sigma$ is a positive friction coefficient. The function $P$ denotes the pressure law and satisfies classical assumptions: the function $P$ is decreasing, convex, and tends to 0 as $\tau \rightarrow \infty$. Denoting $\mathcal{P}$ a primitive form of $P$, the homogeneous $p$-system

$$
\left\{\begin{array}{l}
\varepsilon \partial_{t} \tau-\partial_{x} u=0, \\
\varepsilon \partial_{t} u+\partial_{x} P(\tau)=0
\end{array}\right.
$$

admits an entropy $\eta$ defined by $\eta(\tau, u)=\frac{u^{2}}{2}-\mathcal{P}(\tau)$.

Remark 1.1. For $\tau_{\star}$ fixed in $(0,+\infty)$, the strictly convex function

$$
\eta_{\tau_{\star}}(u, \tau)=\frac{u^{2}}{2}-\left(\mathcal{P}(\tau)-\mathcal{P}\left(\tau_{\star}\right)\right)+P\left(\tau_{\star}\right)\left(\tau-\tau_{\star}\right)
$$

is also an entropy for the system (1) (one could also use the term of relative entropy) and satisfies

$$
\eta_{\tau_{\star}}(u, \tau) \geq 0, \quad \text { and } \quad \eta_{\tau_{\star}}\left(0, \tau^{\star}\right)=0 .
$$

The associated limit model, once again obtained by a Chapman-Enskog expansion, is

$$
\left\{\begin{array}{l}
\partial_{t} \tau+\frac{1}{\sigma} \partial_{x x} P(\tau)=0, \\
u=0 .
\end{array}\right.
$$

Since the pressure law $P$ is a decreasing function, the first equation is a nonlinear parabolic equation.

\section{Asymptotic PRESERVing SCHEMES}

The aim of this part is to present the design of numerical schemes which are compatible with the asymptotic limits of the previous section, which commonly are called asymptotic preserving schemes $[9,14,15,19,20]$.

Definition 2.1. A numerical scheme for system $\left(\mathcal{M}_{f}^{G T}\right)$ (respectively $\left(\mathcal{M}_{f}^{p-s y s}\right)$ ) is said to be asymptotic preserving if it is stable and consistent with the solutions of the hyperbolic model $\left(\mathcal{M}_{f}^{G T}\right)$ (resp. $\left.\left(\mathcal{M}_{f}^{p-s y s}\right)\right)$ for all $\varepsilon>0$ and if, at the limit $\varepsilon \rightarrow 0$, it converges to a stable and consistent numerical scheme with the solutions of the limit parabolic model $\left(\mathcal{M}_{c}^{G T}\right)$ (resp. $\left(\mathcal{M}_{c}^{p-s y s}\right)$ ). 
One of the important points is that the CFL condition for the approximation of the fine model becomes a CFL condition for the approximation of the coarse model. In our case, since we only are dealing with explicit schemes, this means that this condition for $\varepsilon>0$ is of the form $\Delta t \leq C \Delta x$ while at the limit $\varepsilon \rightarrow 0$, it is of the form $\Delta t \leq C \Delta x^{2}$.

In both cases, we construct first a well-balanced scheme, following [16] and [13]. We see that the CFL condition induced by a classical stability analysis is too restrictive to be valid for all $\varepsilon>0$. Therefore, we propose an implicit version of this scheme in order to make it asymptotic preserving. Note that this new scheme is explicit in practice (the matrix to invert actually is diagonal). We then recover the pioneer work of Gosse and Toscani $[14,15]$ in the case of the Goldstein-Taylor and combining their method with a HLL (or equivalently relaxation) scheme, we easily extend it to the nonlinear case of the $p$-system. In both cases, we provide energy or entropy estimates together with CFL conditions and we carefully examine the behavior of these CFL conditions as $\varepsilon \rightarrow 0$.

\subsection{The Goldstein-Taylor model}

We first present the well-balanced scheme for the Goldstein-Taylor model and show that it is not asymptotic preserving. We then propose an implicit modification to correct it, recovering the Gosse-Toscani scheme [14,15].

\subsubsection{The well-balanced scheme}

The scheme of [6] and the scheme of [3] are both based on the construction of well-balanced scheme, following [16] and [13]. As a consequence, applied to system $\left(\mathcal{M}_{f}^{G T}\right)$, these schemes are identical and the authors of these works claim that this scheme is asymptotic preserving. Their analysis is based on arguments of consistency and on numerical tests, because the models they investigate are nonlinear systems and a complete analysis of stability seems to be out of reach.

Actually, this scheme has been initially introduced in [14] in the context of system $\left(\mathcal{M}_{f}^{G T}\right)$. Since this system is linear, a complete analysis of the scheme can be carried out and the conclusion is that this scheme is not asymptotic preserving in the sense of definition 2.1 .

In the sequel, $\Delta x, \Delta t$ denote some positive parameters corresponding respectively to the space and time steps. Let $u_{0}, v_{0} \in L^{2}(\mathbb{R})$ be initial data, then we discretize them as

$$
u_{i}^{0}=\frac{1}{\Delta x} \int_{(i-1 / 2) \Delta x}^{(i+1 / 2) \Delta x} u_{0}(x) \mathrm{d} x, \quad v_{i}^{0}=\frac{1}{\Delta x} \int_{(i-1 / 2) \Delta x}^{(i+1 / 2) \Delta x} v_{0}(x) \mathrm{d} x, \quad \forall i \in \mathbb{Z} .
$$

Thanks to Jensen's inequality, $\left(u_{i}^{0}\right)_{i}$ and $\left(v_{i}^{0}\right)_{i}$ belong to $\ell^{2}(\mathbb{Z})$ and

$$
\left\|\left(u_{i}^{0}\right)_{i}\right\|_{\ell^{2}(\mathbb{Z})} \leq\left\|u_{0}\right\|_{L^{2}(\mathbb{R})}, \quad\left\|\left(v_{i}^{0}\right)_{i}\right\|_{\ell^{2}(\mathbb{Z})} \leq\left\|v_{0}\right\|_{L^{2}(\mathbb{R})} .
$$

For $i \in \mathbb{Z}$ and $n \in \mathbb{N}^{*}$, we denote by $W_{i}^{n}=\left(u_{i}^{n}, v_{i}^{n}\right)^{T}$ the discrete unknowns of the well-balanced finite volume scheme. The derivation of such a scheme, which is described in the works cited above, is based on the introduction of the function $\chi(t, x)=x$. System $\left(\mathcal{M}_{f}^{G T}\right)$ is replaced by the fully first-order system

$$
\left\{\begin{array}{l}
\varepsilon \partial_{t} v+\partial_{x} u=0, \\
\varepsilon \partial_{t} u+a^{2} \partial_{x} v+\frac{\sigma}{\varepsilon} u \partial_{x} \chi=0 \\
\partial_{t} \chi=0
\end{array}\right.
$$

and the associated Riemann problem can be solved, with $\chi_{r}-\chi_{l}=\Delta x$ in order to mimic the jump of $\chi$ at each interface of the mesh. The eigenvalues of this system are 0 and $\pm a / \varepsilon$ and the two intermediate states are 
$\left(\bar{v}^{-}, \bar{u} / K_{\varepsilon}, \chi_{l}\right)$ for $-a / \varepsilon<x / t<0$ and $\left(\bar{v}^{+}, \bar{u} / K_{\varepsilon}, \chi_{r}\right)$ for $0<x / t<a / \varepsilon$, where $K_{\varepsilon}=1+\frac{\sigma \Delta x}{2 a \varepsilon}$ and

$$
\begin{aligned}
\bar{u}\left(W_{l}, W_{r}\right) & =\frac{u_{l}+u_{r}}{2}-\frac{a}{2}\left(v_{r}-v_{l}\right), \\
\bar{v}^{-}\left(W_{l}, W_{r}\right) & =v_{l}-\frac{1}{a}\left(\bar{u}\left(W_{l}, W_{r}\right) / K_{\varepsilon}-u_{l}\right), \\
\bar{v}^{+}\left(W_{l}, W_{r}\right) & =v_{r}+\frac{1}{a}\left(\bar{u}\left(W_{l}, W_{r}\right) / K_{\varepsilon}-u_{r}\right) .
\end{aligned}
$$

As a result, the well-balanced finite volume scheme writes

$$
\begin{aligned}
& v_{i}^{n+1}=v_{i}^{n}-\frac{\Delta t}{\varepsilon K_{\varepsilon} \Delta x}\left[\bar{u}\left(W_{i}^{n}, W_{i+1}^{n}\right)-\bar{u}\left(W_{i-1}^{n}, W_{i}^{n}\right)\right], \\
& u_{i}^{n+1}=u_{i}^{n}-\frac{a^{2} \Delta t}{\varepsilon \Delta x}\left[\bar{v}^{-}\left(W_{i}^{n}, W_{i+1}^{n}\right)-\bar{v}^{+}\left(W_{i-1}^{n}, W_{i}^{n}\right)\right] .
\end{aligned}
$$

Equation (5) can also be written as

$$
u_{i}^{n+1}=u_{i}^{n}-\frac{a^{2} \Delta t}{\varepsilon K_{\varepsilon} \Delta x}\left[\bar{v}\left(W_{i}^{n}, W_{i+1}^{n}\right)-\bar{v}\left(W_{i-1}^{n}, W_{i}^{n}\right)\right]-\frac{\sigma \Delta t}{\varepsilon^{2} K_{\varepsilon}} u_{i}^{n},
$$

where

$$
\bar{v}\left(W_{l}, W_{r}\right)=\frac{v_{l}+v_{r}}{2}-\frac{1}{2 a}\left(u_{r}-u_{l}\right) .
$$

Besides, the scheme (4) for the discretization of $v$ writes

$$
v_{i}^{n+1}=v_{i}^{n}-\frac{\Delta t}{\varepsilon K_{\varepsilon} \Delta x}\left[\frac{u_{i+1}^{n}-u_{i-1}^{n}}{2}+\frac{a}{2}\left(2 v_{i}^{n}-v_{i+1}^{n}-v_{i-1}^{n}\right)\right] .
$$

Proposition 2.1. Under the CFL condition

$$
\frac{1}{\varepsilon+\frac{\sigma \Delta x}{2 a}}\left(\frac{a \Delta t}{\Delta x}+\frac{\sigma \Delta t}{2 \varepsilon\left(\varepsilon+\frac{\sigma \Delta x}{2 a}\right)}\right) \leq 1
$$

the scheme (6)-(8) is $L^{2}$-diminishing, i.e. for all $n \in \mathbb{N}$,

$$
\sum_{i \in \mathbb{Z}} \Delta x\left(\left(u_{i}^{n+1}\right)^{2}+\left(v_{i}^{n+1}\right)^{2}\right) \leq \sum_{i \in \mathbb{Z}} \Delta x\left(\left(u_{i}^{n}\right)^{2}+\left(v_{i}^{n}\right)^{2}\right) .
$$

Proof. In order to prove stability of our scheme under a given CFL condition, we adopt the Von Neumann analysis. Assuming the solution is $L^{2}$, we can introduce its Fourier modes in the following manner:

$$
u^{n}(x)=\sum_{k \in \mathbb{Z}} \hat{u}^{n}(k) e^{i 2 \pi k x} .
$$

Hence,

$$
u^{n}(x+\Delta x)=u^{n}(x) e^{i 2 \pi k \Delta x}
$$

and the application of the Fourier transform on (6)-(8) leads to:

$$
\left(\begin{array}{c}
\hat{u}^{n+1}(k) \\
\hat{v}^{n+1}(k)
\end{array}\right)=\mathbb{A}_{\exp }(k)\left(\begin{array}{c}
\hat{u}^{n}(k) \\
\hat{v}^{n}(k)
\end{array}\right)
$$


where, denoting by

$$
\lambda:=\frac{a \Delta t}{\varepsilon K_{\varepsilon} \Delta x}, \quad \mu:=\frac{\sigma \Delta t}{\varepsilon^{2} K_{\varepsilon}}, \quad \alpha_{k}:=k \pi \Delta x,
$$

the amplification matrix $\mathbb{A}_{\exp }(k)$ can be written

$$
\mathbb{A}_{\exp }(k):=\left(\begin{array}{cc}
1-2 \lambda \sin ^{2}\left(\alpha_{k}\right)-\mu & -i \lambda a \sin \left(2 \alpha_{k}\right) \\
-i \frac{\lambda}{a} \sin \left(2 \alpha_{k}\right) & 1-2 \lambda \sin ^{2}\left(\alpha_{k}\right)
\end{array}\right) .
$$

Define

$$
\delta^{\prime}:=\frac{\mu^{2}}{4}-\lambda^{2} \sin ^{2}\left(2 \alpha_{k}\right),
$$

then the eigenvalues $\nu_{ \pm} \in \mathbb{C}$ of $\mathbb{A}_{\exp }(k)$ are given by

$$
\nu_{ \pm}:=1-2 \lambda \sin ^{2}\left(\alpha_{k}\right)-\frac{\mu}{2} \pm \sqrt{\delta^{\prime}} .
$$

Assume first that $\delta^{\prime} \geq 0$ (this is the case for $\varepsilon$ large enough). In this case, one has $\left|\delta^{\prime}\right| \leq \frac{\mu}{2}$ then $\nu_{+} \leq 1$, while

$$
\nu_{-} \geq 1-2 \lambda-\mu \text {. }
$$

One obtains thus that under the CFL condition (9), one has $\nu_{-} \geq-1$, ensuring the $L^{2}$ stability of the scheme. Assume now that $\delta^{\prime}<0$, so that

$$
\begin{aligned}
\left|\nu_{+}\right|^{2} & =\left|\nu_{-}\right|^{2}=\left(1-2 \lambda \sin ^{2}\left(\alpha_{k}\right)-\frac{\mu}{2}\right)^{2}+\lambda^{2} \sin ^{2}\left(2 \alpha_{k}\right)-\frac{\mu^{2}}{4} \\
& \leq 1+4 \lambda \sin ^{2}\left(\alpha_{k}\right)\left(\lambda+\frac{\mu}{2}-1\right) .
\end{aligned}
$$

Hence, the scheme is $L^{2}$-diminishing under the CFL condition (9).

It particularly follows from (9) that $\Delta t$ has to fulfill the $\varepsilon$-dependent estimate

$$
\Delta t \leq \frac{2 \varepsilon\left(\varepsilon+\frac{\sigma \Delta x}{2 a}\right)}{\sigma} .
$$

Hence, for a fixed space step $\Delta x$, letting $\varepsilon$ tend to 0 requires the time step $\Delta t$ to tend also to 0 . This can also be remarked by taking constant initial data. Using the form (8)-(6), one can directly see that this numerical scheme becomes a modified explicit Euler method for the equation $\varepsilon \partial_{t} u=-(\sigma / \varepsilon) u$. It is well-known that such a method can only be stable under a restrictive condition on the time step $\Delta t$, which makes it tend to 0 when $\varepsilon \rightarrow 0$.

\subsubsection{Implicit modification of the well-balanced scheme}

As mentioned above, the time step $\Delta t$ tends to zero when $\varepsilon$ tends to 0 . The simple, but crucial, idea is then to take an implicit discretization of the source term, as done in [14] (see also [15]). This leads to the new numerical scheme

$$
\begin{aligned}
& u_{i}^{n+1}=u_{i}^{n}-\frac{\Delta t}{\varepsilon K_{\varepsilon} \Delta x}\left[a^{2} \frac{v_{i+1}^{n}-v_{i-1}^{n}}{2}+\frac{a}{2}\left(2 u_{i}^{n}-u_{i+1}^{n}-u_{i-1}^{n}\right)\right]-\frac{\sigma \Delta t}{\varepsilon^{2} K_{\varepsilon}} u_{i}^{n+1}, \\
& v_{i}^{n+1}=v_{i}^{n}-\frac{\Delta t}{\varepsilon K_{\varepsilon} \Delta x}\left[\frac{u_{i+1}^{n}-u_{i-1}^{n}}{2}+\frac{a}{2}\left(2 v_{i}^{n}-v_{i+1}^{n}-v_{i-1}^{n}\right)\right] .
\end{aligned}
$$

The purpose of the following proposition is to show that the new scheme (11)-(12) is $L^{2}$-diminishing for a choice of $\Delta t$ depending on $\Delta x$ but not on $\varepsilon$. 
Proposition 2.2. Under the CFL condition

$$
\frac{1}{\varepsilon+\frac{\sigma \Delta x}{2 a}} \frac{a \Delta t}{\Delta x} \leq 1
$$

the scheme (11), (12) is $L^{2}$-diminishing in the sense of (10). The inequality (13) holds for all $\varepsilon>0$ if $\Delta t$ satisfies the usual parabolic stability condition

$$
\Delta t \leq \frac{\sigma}{2 a^{2}} \Delta x^{2}
$$

which does not depend on $\varepsilon$.

Proof. The scheme (11)-(12) can be obtained by splitting the convection and the source term, i.e.

- step 1: compute the convective contribution, i.e.

$$
\begin{aligned}
& u_{i}^{n+1 / 2}=u_{i}^{n}-\frac{\Delta t}{\varepsilon K_{\varepsilon} \Delta x}\left[a^{2} \frac{v_{i+1}^{n}-v_{i-1}^{n}}{2}+\frac{a}{2}\left(2 u_{i}^{n}-u_{i+1}^{n}-u_{i-1}^{n}\right)\right], \\
& v_{i}^{n+1 / 2}=v_{i}^{n}-\frac{\Delta t}{\varepsilon K_{\varepsilon} \Delta x}\left[\frac{u_{i+1}^{n}-u_{i-1}^{n}}{2}+\frac{a}{2}\left(2 v_{i}^{n}-v_{i+1}^{n}-v_{i-1}^{n}\right)\right]
\end{aligned}
$$

- step 2: take the reaction term into account, i.e.

$$
u_{i}^{n+1}\left(1+\frac{\sigma \Delta t}{\varepsilon^{2} K_{\varepsilon}}\right)=u_{i}^{n+1 / 2}, \quad v_{i}^{n+1}=v_{i}^{n+1 / 2} .
$$

Clearly, the step 1 is $L^{2}$-diminishing under the CFL condition (13). Indeed, the scheme (15), (16) corresponds to the former scheme (6)-(8) in the particular case where $\sigma=0$. Therefore, its stability follows from Proposition 2.1. The step 2 is obviously $L^{2}$-diminishing since for all $i,\left(u_{i}^{n+1}\right)^{2} \leq\left(u_{i}^{n+1 / 2}\right)^{2}$.

We establish now that the scheme (11)-(12) is asymptotic preserving, in the sense that, for fixed $\Delta x$ and for a convenient choice of $\Delta t$, then, letting $\varepsilon$ tend to 0 , the solution $W_{i}^{\varepsilon, n+1}=\left(u_{i}^{\varepsilon, n+1}, v_{i}^{\varepsilon, n+1}\right)^{T}$ of $(11)$ (12) tends towards $W_{i}^{0, n+1}$ which is the solution of a finite volume scheme which is consistent with the limit problem $\left(\mathcal{M}_{c}^{G T}\right)$.

Proposition 2.3. Let $\left(W_{i}^{\varepsilon, n+1}\right)_{i \in \mathbb{Z}, n \in \mathbb{N}}$ be the solution of (11)-(12) corresponding to data $\left(u_{i}^{0}\right)_{i \in \mathbb{Z}}$ and $\left(v_{i}^{0}\right)_{i \in \mathbb{Z}}$ belonging to $\ell^{2}(\mathbb{Z})$. Assume that $(14)$ holds, then, for all $i \in \mathbb{Z}, u_{i}^{\varepsilon, n+1}$ tends to 0 as $\varepsilon \rightarrow 0$ and $v_{i}^{\varepsilon, n+1}$ tends towards the solution $v_{i}^{0, n+1}$ of the consistent discretization of $\left(\mathcal{M}_{c}^{G T}\right)$ given by

$$
\begin{aligned}
v_{i}^{0, n+1} & =v_{i}^{n}-\frac{a^{2} \Delta t}{\sigma \Delta x^{2}}\left[2 v_{i}^{n}-v_{i-1}^{n}-v_{i+1}^{n}\right], \quad \text { if } n \geq 1, \\
v_{i}^{0,1} & =v_{i}^{0}-\frac{a^{2} \Delta t}{\sigma \Delta x^{2}}\left[2 v_{i}^{0}-v_{i-1}^{0}-v_{i+1}^{0}\right]-\frac{a \Delta t}{\sigma \Delta x^{2}}\left[u_{i+1}^{0}-u_{i-1}^{0}\right] .
\end{aligned}
$$

As a consequence, the numerical scheme (11)-(12) under the CFL condition (13) is asymptotic preserving in the sense of definition 2.1 .

Proof. Assume that (14) holds, so that it follows from the proof of Proposition 2.2 that for all $\varepsilon$ belonging to an unlabeled sequence of $\mathbb{R}_{+}^{*}$ tending to 0 ,

$$
\sum_{i \in \mathbb{Z}} \Delta x\left(\left(u_{i}^{\varepsilon, n+1 / 2}\right)^{2}+\left(v_{i}^{\varepsilon, n+1}\right)^{2}\right) \leq \sum_{i \in \mathbb{Z}} \Delta x\left(\left(u_{i}^{0}\right)^{2}+\left(v_{i}^{0}\right)^{2}\right)<+\infty,
$$


where $u_{i}^{\varepsilon, n+1 / 2}$ is given by (15). Since $\Delta x$ is fixed, we know that there exists $C \geq 0$ not depending on $\varepsilon$ such that

$$
\forall i \in \mathbb{Z}, \quad\left|u_{i}^{\varepsilon, n+1 / 2}\right|,\left|v_{i}^{\varepsilon, n+1}\right| \leq C .
$$

Therefore, since $\sigma>0$ and since $\varepsilon^{2} K_{\varepsilon} \rightarrow 0$ as $\varepsilon \rightarrow 0$, it follows from (17) that $u_{i}^{\varepsilon, n+1} \rightarrow 0$ as $\varepsilon \rightarrow 0$. Thanks to (20), there exists an unlabeled subsequence of $\left(v_{i}^{\varepsilon, n+1}\right)_{\varepsilon}$ converging towards some $v_{i}^{0, n+1}$ with $\left(v_{i}^{0, n+1}\right)_{i} \in \ell^{2}(\mathbb{Z})$. Using the fact that $\varepsilon K_{\varepsilon} \rightarrow \frac{\sigma \Delta x}{2 a}$, we obtain that (18) and (19) are fulfilled at the limit $\varepsilon \rightarrow 0$.

Remark 2.1. As it appears in the first iteration of the limit scheme (19), an additional quantity

$$
r_{i}^{0}:=\frac{a \Delta t}{\sigma \Delta x^{2}}\left[u_{i-1}^{0}-u_{i+1}^{0}\right]
$$

has to be taken in consideration. It is easy to check that for $u_{0} \in L^{2}(\mathbb{R})$, thanks to (14), the quantity $r_{i}^{0}$ tends to 0 in $\ell^{2}(\mathbb{Z})$ as $\Delta x, \Delta t \rightarrow 0$. More precisely, if $u_{0} \in H^{1}(\mathbb{R})$, there exists $C$ depending neither on $\Delta x$ nor on $\Delta t$ such that $\left\|\left(r_{i}^{0}\right)\right\|_{\ell^{2}(\mathbb{Z})} \leq C \Delta x$. Denote by

$$
\mathcal{A}:\left\{\begin{aligned}
\ell^{2}(\mathbb{Z}) & \rightarrow \ell^{2}(\mathbb{Z}) \\
w_{i} & \mapsto w_{i}-\frac{a^{2} \Delta t}{\sigma \Delta x^{2}}\left[2 w_{i}-w_{i-1}-w_{i+1}\right]
\end{aligned}\right.
$$

and assume that there exists $\zeta \in(0,1)$ such that the following more restrictive $C F L$ condition holds: $\frac{4 a^{2} \Delta t}{\sigma \Delta x^{2}} \leq \zeta$. Then the mapping $\mathcal{A}$ is invertible and $\left\|\mathcal{A}^{-1}\right\| \leq \frac{1}{1-\zeta}$. Therefore, there exists a unique $\left(\tilde{v}_{i}^{0}\right) \in \ell^{2}(\mathbb{Z})$, such that $\mathcal{A} \tilde{v}_{i}^{0}=r_{i}^{0}$. Moreover, $\left(\tilde{v}_{i}^{0}\right)$ tends to 0 in $\ell^{2}(\mathbb{Z})$ as $\Delta t, \Delta x \rightarrow 0$ since $\left(r_{i}^{0}\right)$ does. The solution $\left(v_{i}^{n}\right)_{i, n}$ of the scheme (18)-(19) is then the solution of the usual approximation of the heat equation (18) for the slightly modified initial data $v_{i}^{0}+\tilde{v}_{i}^{0}$, the correction $\left(\tilde{v}_{i}^{0}\right)_{i \in \mathbb{Z}}$ tending to zero with the discretization parameters $\Delta x, \Delta t$. Thanks to the continuity of the solution $v$ to the heat equation with respect to the initial data $v_{0}$, this allows to claim that the discrete solution provided by the scheme (18)-(19) converges in $L^{2}(\mathbb{R})$ towards the unique solution $v$ of the heat equation

$$
\partial_{t} v-\frac{a^{2}}{\sigma} \partial_{x x} v=0, \quad v_{\left.\right|_{t=0}}=v_{0} .
$$

\subsection{The $p$-system}

Let us now consider the case of the $p$-system.

\subsubsection{The well-balanced scheme}

Once again, the schemes proposed in [6] and in [3] follow the construction of well-balanced schemes and lead to the same numerical scheme. It is based on the HLL scheme [17] and can also be derived by the mean of a relaxation procedure (see e.g. $[4,6,7]$ ). It writes, for all $i \in \mathbb{Z}$ and $n \in \mathbb{N}$,

$$
\begin{aligned}
& \tau_{i}^{n+1}=\tau_{i}^{n}-\frac{\Delta t}{\varepsilon \Delta x}\left[u_{i-1 / 2}^{n}-u_{i+1 / 2}^{n}\right], \\
& u_{i}^{n+1}=u_{i}^{n}-\frac{\Delta t}{\varepsilon \Delta x}\left[\Pi_{i+1 / 2}^{n,-}-\Pi_{i-1 / 2}^{n,-}\right]
\end{aligned}
$$


where $u_{i+1 / 2}^{n}, \Pi_{i+1 / 2}^{n, \pm}$ and $\tau_{i+1 / 2}^{n, \pm}$ are given by

$$
\begin{aligned}
u_{i+1 / 2}^{n} & =\frac{1}{1+\frac{\sigma \Delta x}{2 \varepsilon a}}\left[\frac{u_{i}^{n}+u_{i+1}^{n}}{2}+\frac{P\left(\tau_{i}^{n}\right)-P\left(\tau_{i+1}^{n}\right)}{2 a}\right], \\
\Pi_{i+1 / 2}^{n,-} & =P\left(\tau_{i}^{n}\right)-a\left(u_{i+1 / 2}^{n}-u_{i}^{n}\right), \\
\Pi_{i+1 / 2}^{n,+} & =P\left(\tau_{i+1}^{n}\right)-a\left(u_{i+1}^{n}-u_{i+1 / 2}^{n}\right) .
\end{aligned}
$$

In the above system, $a>0$ is a parameter to be fixed later (cf. Proposition 2.4). By taking (22)-(24) in consideration in (21) and introducing $L_{\varepsilon}=1+\frac{\sigma \Delta x}{2 \varepsilon a}$, this scheme can be reformulated as

$$
\begin{aligned}
& \tau_{i}^{n+1}=\tau_{i}^{n}-\frac{\Delta t}{\varepsilon L_{\varepsilon} \Delta x}\left[\frac{u_{i-1}^{n}-u_{i+1}^{n}}{2}+\frac{1}{2 a}\left(-2 P\left(\tau_{i}^{n}\right)+P\left(\tau_{i+1}^{n}\right)+P\left(\tau_{i-1}^{n}\right)\right)\right], \\
& u_{i}^{n+1}=u_{i}^{n}-\frac{\Delta t}{\varepsilon L_{\varepsilon} \Delta x}\left[\frac{P\left(\tau_{i+1}^{n}\right)-P\left(\tau_{i-1}^{n}\right)}{2}+\frac{a}{2}\left(2 u_{i}^{n}-u_{i-1}^{n}-u_{i+1}^{n}\right)\right]-\frac{\sigma \Delta t}{\varepsilon^{2} L_{\varepsilon}} u_{i}^{n} .
\end{aligned}
$$

We fail to obtain a complete analysis of stability for the scheme (25), (26). Nonetheless, a simple case can be used to check that this numerical scheme also suffer from a too restrictive stability condition of the same type as (9). Assume that

$$
\forall i \in \mathbb{Z} \quad \tau_{i}^{n}=\bar{\tau}, u_{i}^{n}=\bar{u},
$$

where $\bar{\tau}$ and $\bar{u}$ are two positive constant. Then, the scheme (25)-(26) writes

$$
\forall i \in \mathbb{Z}, \quad \tau_{i}^{n+1}=\bar{\tau}, \quad u_{i}^{n+1}=\left(1-\frac{\sigma \Delta t}{\varepsilon^{2} L_{\varepsilon}}\right) \bar{u} .
$$

This formula corresponds to an explicit discretization of the ordinary differential equation

$$
\left\{\begin{array}{l}
\tau^{\prime}(t)=0 \\
u^{\prime}(t)=-\frac{\sigma}{\varepsilon} u
\end{array}\right.
$$

As a consequence, a necessary condition of the stability (in $L^{2}$ for instance) of the scheme is

$$
\frac{\sigma \Delta t}{2 \varepsilon^{2} L_{\varepsilon}} \leq 1
$$

Here again, this condition implies that $\Delta t \rightarrow 0$ when $\varepsilon \rightarrow 0$, which means that the scheme (21) is not asymptotic preserving.

\subsubsection{Implicit modification of the well-balanced scheme}

Following the idea used for the Goldstein-Taylor system, we replace the explicit discretization of the source term of (26) by an implicit one, which yields

$$
\begin{aligned}
& \tau_{i}^{n+1}=\tau_{i}^{n}-\frac{\Delta t}{\varepsilon L_{\varepsilon} \Delta x}\left[\frac{u_{i-1}^{n}-u_{i+1}^{n}}{2}+\frac{1}{2 a}\left(-2 P\left(\tau_{i}^{n}\right)+P\left(\tau_{i+1}^{n}\right)+P\left(\tau_{i-1}^{n}\right)\right)\right], \\
& u_{i}^{n+1}=u_{i}^{n}-\frac{\Delta t}{\varepsilon L_{\varepsilon} \Delta x}\left[\frac{P\left(\tau_{i+1}^{n}\right)-P\left(\tau_{i-1}^{n}\right)}{2}+\frac{a}{2}\left(2 u_{i}^{n}-u_{i-1}^{n}-u_{i+1}^{n}\right)\right]-\frac{\sigma \Delta t}{\varepsilon^{2} L_{\varepsilon}} u_{i}^{n+1} .
\end{aligned}
$$

While we have not been able to completely study the stability of the numerical scheme (21) (but we proved that the associated CFL condition is too restrictive), we can prove that this new scheme is stable under an 
$\varepsilon$-independent CFL condition and therefore, it is asymptotic preserving. Note that the stability we invoke is the decrease of the entropy.

Let $\tau_{\star} \in(0, \infty)$, let $\eta_{\tau_{\star}}$ be the entropy of $(1)$ defined by $(2)$, and let $\tau_{0}, u_{0}$ be measurable functions such that

$$
\int_{\mathbb{R}} \eta_{\tau_{\star}}\left(\tau_{0}, u_{0}\right) \mathrm{d} x<+\infty
$$

and such that $\left(\tau_{0}-\tau_{\star}\right) \in L^{\infty} \cap L^{1}(\mathbb{R})$. We moreover assume that $\tau_{0}$ is essentially bounded away from 0 . The initial data $\tau_{0}, u_{0}$ are discretized by

$$
\tau_{i}^{0}=\frac{1}{\Delta x} \int_{(i-1 / 2) \Delta x}^{(i+1 / 2) \Delta x} \tau_{0}(x) \mathrm{d} x, \quad u_{i}^{0}=\frac{1}{\Delta x} \int_{(i-1 / 2) \Delta x}^{(i+1 / 2) \Delta x} u_{0}(x) \mathrm{d} x,
$$

so that, thanks to Jensen inequality,

$$
\sum_{i \in \mathbb{Z}} \eta_{\tau_{\star}}\left(\tau_{i}^{0}, u_{i}^{0}\right) \Delta x \leq \int_{\mathbb{R}} \eta_{\tau_{\star}}\left(\tau_{0}, u_{0}\right) \mathrm{d} x<+\infty .
$$

In particular, there exists $\underline{\tau}, \bar{\tau}>0$ such that $0<\underline{\tau} \leq \tau_{\star} \leq \bar{\tau}<\infty$, and such that $\underline{\tau} \leq \tau_{0} \leq \bar{\tau}$ a.e. in $\mathbb{R}$.

Proposition 2.4. Under the Whitham condition

$$
a^{2} \geq 2 \max _{n \in \mathbb{N}} \max _{i \in \mathbb{Z}}\left(-P^{\prime}\left(\tau_{i}^{n}\right),-P^{\prime}\left(\tau_{i+1 / 2}^{n, \pm}\right)\right)
$$

where the quantities $\tau_{i+1 / 2}^{n, \pm}$ are defined by

$$
\tau_{i+1 / 2}^{n,-}=\tau_{i}^{n}+\frac{1}{a}\left(u_{i+1 / 2}^{n}-u_{i}^{n}\right), \quad \tau_{i+1 / 2}^{n,+}=\tau_{i+1}^{n}+\frac{1}{a}\left(u_{i+1}^{n}-u_{i+1 / 2}^{n}\right),
$$

and the CFL condition

$$
\frac{2 a \Delta t}{\Delta x\left(\varepsilon+\frac{\sigma \Delta x}{2 a}\right)} \leq 1
$$

then the scheme is entropy dissipative, i.e. the solution $\left(\tau_{i}^{n+1}, u_{i}^{n+1}\right)_{i \in \mathbb{Z}}$ of the scheme (27), (28) satisfies

$$
\sum_{i \in \mathbb{Z}} \eta_{\tau_{\star}}\left(\tau_{i}^{n+1}, u_{i}^{n+1}\right) \Delta x \leq \sum_{i \in \mathbb{Z}} \eta_{\tau_{\star}}\left(\tau_{i}^{n}, u_{i}^{n}\right) \Delta x
$$

Proof. As previously in the proof of Proposition 2.2, making the source term implicit allows to write the scheme (27), (28) as a splitting scheme.

- Step 1. We compute the convective contribution given by:

$$
\begin{aligned}
& \tau_{i}^{n+1 / 2}=\tau_{i}^{n}-\frac{\Delta t}{\varepsilon L_{\varepsilon} \Delta x}\left[\frac{u_{i-1}^{n}-u_{i+1}^{n}}{2}+\frac{1}{2 a}\left(-2 P\left(\tau_{i}^{n}\right)+P\left(\tau_{i+1}^{n}\right)+P\left(\tau_{i-1}^{n}\right)\right)\right], \\
& u_{i}^{n+1 / 2}=u_{i}^{n}-\frac{\Delta t}{\varepsilon L_{\varepsilon} \Delta x}\left[\frac{P\left(\tau_{i+1}^{n}\right)-P\left(\tau_{i-1}^{n}\right)}{2}+\frac{a}{2}\left(2 u_{i}^{n}-u_{i-1}^{n}-u_{i+1}^{n}\right)\right] .
\end{aligned}
$$

This scheme is nothing but the usual HLL scheme to solve

$$
\partial_{t} \tau-\partial_{x} u=0, \quad \partial_{t} u+\partial_{x} P(\tau)=0
$$

with a time step $\overline{\Delta t}=\frac{\Delta t}{\varepsilon L_{\varepsilon}}$. Following [4], this scheme is entropy dissipative under the Whitham condition (30) and under the CFL condition (31). 
- Step 2. The contribution of the source term is now computed with the implicit scheme

$$
\tau_{i}^{n+1}=\tau_{i}^{n+1 / 2}, \quad u_{i}^{n+1}=\left(1+\frac{\sigma \Delta t}{\varepsilon^{2} L_{\varepsilon}}\right)^{-1} u_{i}^{n+1 / 2} .
$$

In view of the definition (2) of the entropy $\eta_{\tau_{\star}}$, this step is also entropy dissipative, so that the relation (32) holds.

Remark 2.2. As it was noticed in Proposition 2.2 for the case of the Goldstein-Taylor model, the CFL condition (31) is fulfilled for all $\varepsilon>0$ if one requires that

$$
\Delta t \leq \frac{\sigma \Delta x^{2}}{4 a^{2}}
$$

As done previously on the Goldstein-Taylor model, we investigate the limit as $\varepsilon$ tends to 0 for fixed discretization parameters of the discrete solution, denoted by $\left(\tau_{i}^{\varepsilon, n}, u_{i}^{\varepsilon, n}\right)_{i, n}$, of the scheme (27)-(28).

Proposition 2.5. Under the Whitham condition (30), the CFL condition (34) and the finite entropy condition (29), then for all $i \in \mathbb{Z}$, the limit of the scheme (27)-(28) when $\varepsilon \rightarrow 0$ writes

$$
\begin{aligned}
\tau_{i}^{0, n+1} & =\tau_{i}^{0, n}-\frac{\Delta t}{\sigma \Delta x^{2}}\left[-2 P\left(\tau_{i}^{0, n}\right)+P\left(\tau_{i-1}^{0, n}\right)+P\left(\tau_{i+1}^{0, n}\right)\right] \quad \forall n \geq 1, \\
\tau_{i}^{0,1} & =\tau_{i}^{0}-\frac{\Delta t}{\sigma \Delta x^{2}}\left[-2 P\left(\tau_{i}^{0}\right)+P\left(\tau_{i-1}^{0}\right)+P\left(\tau_{i+1}^{0}\right)+a\left(u_{i-1}^{0}-u_{i+1}^{0}\right)\right] .
\end{aligned}
$$

Proof. It follows from Proposition 2.4 and Remark 2.2 that under the Whitham condition (30), the CFL condition (34) and the finite entropy condition (29) on the initial data, one has, for all $n \geq 1$,

$$
\sum_{i \in \mathbb{Z}} \Delta x\left[\eta\left(\tau_{i}^{\varepsilon, n}, u_{i}^{\varepsilon, n}\right)\right] \leq \int_{\mathbb{R}} \eta\left(\tau_{0}, u_{0}\right) \mathrm{d} x<\infty .
$$

This ensures that there exists $C \geq 0$ not depending on $\varepsilon$ such that for all $\varepsilon>0$, one has

$$
\forall i \in \mathbb{Z}, \forall n \geq 1, \quad\left|u_{i}^{\varepsilon, n}\right| \leq C, \quad-\mathcal{P}\left(\tau_{i}^{\varepsilon, n}\right) \leq C
$$

Hence, there exists $\left(u_{i}^{0, n}\right)_{i, n}$ and $\left(\mathcal{P}_{i}^{0, n}\right)_{i, n}$ such that up to an unlabeled subsequence,

$$
\forall i \in \mathbb{Z}, \quad u_{i}^{\varepsilon, n} \rightarrow u_{i}^{0}, \quad \mathcal{P}\left(\tau_{i}^{\varepsilon, n}\right) \rightarrow \mathcal{P}_{i}^{0, n} \quad \text { as } \varepsilon \rightarrow 0 .
$$

Since $P$ is strictly increasing and continuous, then, denoting by $\tau_{i}^{0, n}=P^{-1}\left(\mathcal{P}_{i}^{0, n}\right)$, we obtain that

$$
\forall i \in \mathbb{Z}, \quad \tau_{i}^{\varepsilon, n} \rightarrow \tau_{i}^{0, n} \quad \text { as } \varepsilon \rightarrow 0
$$

Thanks to (33), we obtain that for all $n \geq 1$ and for all $i \in \mathbb{Z}, u_{i}^{0, n}=0$. Since $2 a \varepsilon L_{\varepsilon} \rightarrow \sigma \Delta x$ as $\varepsilon \rightarrow 0$ (recall that $L_{\varepsilon}=1+\frac{\sigma \Delta x}{2 \varepsilon a}$ ), considering the limit as $\varepsilon \rightarrow 0$ in (27) yields (35), while a contribution coming from $u_{0}$ still appears in the computation (36) at the first time step. Obviously, since (35)-(36) define $\left(\tau_{i}^{0, n}\right)_{i, n}$ in a unique way, the whole unlabeled sequence $\left(\left(\tau_{i}^{\varepsilon, n}\right)_{i, n}\right)_{\varepsilon}$ converges towards $\left(\tau_{i}^{0, n}\right)_{i, n}$ when $\varepsilon \rightarrow 0$. 
We provide now the proof of convergence of the scheme (35), (36) towards the unique weak solution of the problem

$$
\partial_{t} \tau+\frac{1}{\sigma} \partial_{x x} P(\tau)=0, \quad \tau_{\mid t=0}=\tau_{0}, \quad \lim _{x \rightarrow \pm \infty} \tau=\tau_{\star} .
$$

Lemma 2.6. Assume that $a^{2}>-P^{\prime}(\underline{\tau})$, then there exists $\omega \in(0, \underline{\tau})$ and $\overline{\Delta x}_{\omega}>0$ such that for all $\Delta x \leq \overline{\Delta x}_{\omega}$, one has

$$
\tau_{i}^{0, n} \in[\underline{\tau}-\omega, \underline{\tau}+\omega], \quad \forall i \in \mathbb{Z}, \forall n \geq 1 .
$$

Proof. Since $a^{2}>-P^{\prime}(\underline{\tau})$, there exists $\omega>0$ such that $a^{2}=-P^{\prime}(\underline{\tau}-\omega)$. Denote by $\left(\tilde{\tau}_{i}^{0,1}\right)_{i}$ the quantities defined by

$$
\tilde{\tau}_{i}^{0,1}=\tau_{i}^{0}-\frac{\Delta t}{\sigma \Delta x^{2}}\left[-2 P\left(\tau_{i}^{0}\right)+P\left(\tau_{i-1}^{0}\right)+P\left(\tau_{i+1}^{0}\right)\right], \quad \forall i \in \mathbb{Z} .
$$

Then it follows from classical monotonicity arguments that

$$
\underline{\tau} \leq \tilde{\tau}_{i}^{0,1} \leq \bar{\tau}, \quad \forall i \in \mathbb{Z} .
$$

Now, since

$$
\tau_{i}^{0,1}=\tilde{\tau}_{i}^{0,1}+\frac{a \sigma \Delta t}{\Delta x^{2}}\left(u_{i+1}^{0}-u_{i-1}^{0}\right),
$$

we obtain, thanks to the CFL assumption (34) and from the regularity of $u_{0}$ that for $\Delta x$ small enough,

$$
\tau_{i}^{0,1} \in[\underline{\tau}-\omega, \bar{\tau}+\omega], \quad \forall i \in \mathbb{Z} .
$$

Hence, the condition $a^{2}=-P^{\prime}(\underline{\tau}-\omega)$ ensures that the scheme is still monotone on this new range of values of $\tau_{i}^{0, n}$. Therefore, from a usual discrete maximum principle, we obtain by a straightforward induction that (39) holds.

We now state a $L^{1}$-stability result, whose proof relies on classical monotonicity arguments (see e.g. [8]).

Lemma 2.7. Let $\left(\tau_{i}^{0, n}\right)_{i, n},\left(\check{\tau}_{i}^{0, n}\right)_{i, n}$ be solutions of (35)-(36), corresponding to initial data $\left(\tau_{i}^{0}\right)_{i},\left(\check{\tau}_{i}^{0}\right)_{i} \in$ $[\underline{\tau}, \bar{\tau}]$, then, for $a^{2}>-P^{\prime}(\underline{\tau})$, under the CFL condition (34), and for $\Delta x$ small enough, one has, for all $n \geq 0$,

$$
\sum_{i \in \mathbb{Z}} \Delta x\left|\tau_{i}^{0, n+1}-\check{\tau}_{i}^{0, n+1}\right| \leq \sum_{i \in \mathbb{Z}} \Delta x\left|\tau_{i}^{0, n}-\check{\tau}_{i}^{0, n}\right| .
$$

Denote by $\tau_{\Delta x}$ the piecewise constant function defined almost everywhere by

$$
\tau_{\Delta x}(x, t)=\tau_{i}^{0, n} \quad \text { if }(x, t) \in((i-1 / 2) \Delta x,(i+1 / 2) \Delta x) \times[n \Delta t,(n+1) \Delta t) .
$$

From previous lemma, we deduce directly the following regularity result by choosing $\check{\tau}_{i}^{0}=\tau_{i-1}^{0}$ :

Lemma 2.8. Assume that $\tau_{0} \in L^{\infty}(\mathbb{R} ;[\underline{\tau}, \bar{\tau}])$ with $\tau_{0}-\tau_{\star} \in L^{1}(\mathbb{R})$, assume that $a^{2}>-P^{\prime}(\underline{\tau})$ and that (34) holds, and assume that $\Delta x$ is small enough, then, for all $n \geq 1$,

$$
\sum_{i \in \mathbb{Z}}\left|\tau_{i}^{0, n}-\tau_{i-1}^{0, n}\right| \Delta x \leq \sum_{i \in \mathbb{Z}}\left|\tau_{i}^{0}-\tau_{i-1}^{0}\right| \Delta x+\sum_{i} \frac{a \sigma \Delta t}{\Delta x^{2}}\left|u_{i+1}^{0}-u_{i}^{0}-\left(u_{i-1}^{0}-u_{i-2}^{0}\right)\right| .
$$

In particular, under the CFL assumption (34) and if $\tau_{0} \in B V(\mathbb{R})$ and $u_{0} \in W^{1, \infty} \cap L^{2}(\mathbb{R})$, then there exists $C$ not depending on $\Delta x, \Delta t$ such that

$$
T V\left(\tau_{\Delta x}(\cdot, t)\right) \leq T V\left(u_{0}\right)+C<\infty, \quad \forall t \in \mathbb{R}_{+} .
$$


Assume that the assumptions of Lemma 2.8 hold, then, thanks to the tools introduced in [10], we can claim that, up to an unlabeled subsequence of $\left(\tau_{\Delta x}\right)_{\Delta x}$, then there exists $\tau \in L^{\infty} \cap B V(\mathbb{R})$ that satisfies

$$
\tau_{\Delta x} \rightarrow \tau \quad \text { in } L_{\mathrm{loc}}^{1}\left(\mathbb{R} \times \mathbb{R}_{+}\right) \text {as } \Delta x, \Delta t \rightarrow 0
$$

It remains to check that $\tau$ satisfies (38), in particular that for all $\psi \in \mathcal{D}\left(\mathbb{R} \times \mathbb{R}_{+}\right)$, the following weak formulation holds:

$$
\iint_{\mathbb{R} \times \mathbb{R}_{+}} \tau \partial_{t} \psi \mathrm{d} x \mathrm{~d} t+\int_{\mathbb{R}_{0}} \tau_{0} \psi(\cdot, 0) \mathrm{d} x-\iint_{\mathbb{R} \times \mathbb{R}_{+}} P(\tau) \partial_{x x} \psi \mathrm{d} x \mathrm{~d} t=0
$$

The condition at $x \rightarrow \pm \infty$ is fulfilled in the sense that, as a direct consequence of Lemma 2.7, one has $\tau-\tau_{\star} \in L^{1}(\mathbb{R})$.

Proposition 2.9. Assume that $u_{0} \in W^{1, \infty}(\mathbb{R})$, that $\tau_{0} \in B V(\mathbb{R})$ such that $\tau_{0} \in L^{\infty}(\mathbb{R} ;[\underline{\tau}, \bar{\tau}])$ and $\tau_{0}-\tau_{\star} \in$ $L^{1}(\mathbb{R})$, then, under the condition (34) with $a^{2}>-P^{\prime}(\underline{\tau})$, the limit $\tau$ of $\tau_{\Delta x}$ exhibited in (41) is a solution of (38) in the sense that $\tau-\tau_{\star} \in L^{\infty}\left(\mathbb{R}_{+} ; L^{1}(\mathbb{R})\right)$ and that the weak formulation $(42)$ is satisfied. As a consequence, the numerical scheme (27)-(28) is asymptotic preserving.

Proof. The convergence of $\tau_{\Delta x}$ towards $\tau$ in the good functional space has been already justified from compactness results. It remains to check that the weak formulation (42) is satisfied. Let $\psi \in \mathcal{D}\left(\mathbb{R} \times \mathbb{R}_{+}\right)$, then we denote by

$$
\psi_{i}^{n}=\frac{1}{\Delta x} \int_{(i-1 / 2) \Delta x}^{(i+1 / 2) \Delta x} \psi(x, n \Delta t) \mathrm{d} x, \quad, \forall i \in \mathbb{Z}, \forall n \geq 0 .
$$

Multiplying the scheme $(35)$ by $-\psi_{i}^{n+1} \Delta x$, (36) by $-\psi_{i}^{1} \Delta x$, then summing on $i \in \mathbb{Z}$ and $n \in \mathbb{N}$ yields, after reorganization of the sums,

$$
A+B+C+D=0
$$

where

$$
\begin{aligned}
A & =\sum_{i \in \mathbb{Z}} \sum_{n \geq 0} \tau_{i}^{0, n} \frac{\psi_{i}^{n+1}-\psi_{i}^{n}}{\Delta t} \Delta x \Delta t, \\
B & =\sum_{i \in \mathbb{Z}} \tau_{i}^{0} \psi_{i}^{0} \Delta x \\
C & =\sum_{i \in \mathbb{Z}} \sum_{n \geq 0} P\left(\tau_{i}^{n}\right)\left(\frac{2 \psi_{i}^{n}-\psi_{i+1}^{n}-\psi_{i-1}^{n}}{\Delta x^{2}}\right) \Delta x \Delta t, \\
D & =\sum_{i \in \mathbb{Z}} \frac{a \Delta t}{\sigma \Delta x^{2}}\left(u_{i+1}-u_{i-1}\right) \psi_{i}^{1} \Delta x .
\end{aligned}
$$

It follows from the $L^{1}$ convergence of $\tau_{\Delta x}$ towards $\tau$ and Lemma 2.6 that

$$
\begin{aligned}
\lim _{\Delta t, \Delta x \rightarrow 0} A & =\iint_{\mathbb{R} \times \mathbb{R}_{+}} \tau \partial_{t} \psi \mathrm{d} x \mathrm{~d} t, \\
\lim _{\Delta x \rightarrow 0} B & =\int \tau_{\mathbb{R}} \tau_{0} \psi(\cdot, 0) \mathrm{d} x, \\
\lim _{\Delta t, \Delta x \rightarrow 0} C & =-\iint_{\mathbb{R} \times \mathbb{R}_{+}} P(\tau) \partial_{x x} \psi \mathrm{d} x \mathrm{~d} t .
\end{aligned}
$$

Now, we deduce from the CFL condition (34) and from the regularity of $u_{0}$ that

$$
|D| \leq C \Delta x
$$


Therefore, by considering the limit $\Delta x, \Delta t \rightarrow 0$ in (43), it follows from (44)-(47) that $\tau$ satisfies (42).

\section{Interface COUPLing BetWeEn THE Fine MODEL AND THE COARSE MODEL}

In this section, we investigate the coupling through a thin interface between the hyperbolic model $\left(\mathcal{M}_{f}^{G T}\right)$ or $\left(\mathcal{M}_{f}^{p-s y s}\right)$ with the parabolic model corresponding to its large time asymptotic limit, namely $\left(\mathcal{M}_{c}^{G T}\right)$ or $\left(\mathcal{M}_{c}^{p-s y s}\right)$. As shown later in figure 2 for instance, a naive coupling by transmission of the information by Dirichlet boundary conditions in the spirit of $[11,12]$ leads to inaccurate results. Therefore, we solve at the thin interface a coupled system in order to connect the fluxes of both models.

\subsection{The Goldstein-Taylor model}

Let us first illustrate the construction of the coupling condition on the simplest example, that is the GoldsteinTaylor model. Consider a fixed interface at $x_{I+1 / 2} \in \mathbb{R}$. We aim at giving a numerical value for the fluxes at the interface $x_{I+1 / 2}$ for the variables $u, v$, where, on the left side of the interface, the fine model is solved:

$$
\left\{\begin{array}{l}
\varepsilon \partial_{t} v+\partial_{x} u=0 \\
\varepsilon \partial_{t} u+a^{2} \partial_{x} v=-\frac{\sigma}{\varepsilon} u, \quad \text { for }(x, t) \text { in } \mathcal{D}_{f}=\left(-\infty, x_{I+1 / 2}\right) \times[0, \infty) \\
u(x, 0)=u_{L}, \\
v(x, 0)=v_{L},
\end{array}\right.
$$

while, on the other side of the interface, the coarse model is considered:

$$
\left\{\begin{array}{l}
\partial_{t} v-\frac{a^{2}}{\sigma} \partial_{x x} v=0, \\
u=0, \\
v(x, 0)=v_{R},
\end{array} \quad \text { for }(x, t) \text { in } \mathcal{D}_{c}=\left(x_{I+1 / 2}, \infty\right) \times[0, \infty)\right.
$$

A first naive idea would be to solve the Riemann problem for the fine problem on both side of the interface, with the initial data $u_{R}=0$ and use the solution to construct numerical fluxes for both sides. As illustrated on Figure 2-right, this algorithm produces quite bad results. In this test case, the regime is such that the GoldsteinTaylor model $\left(\mathcal{M}_{f}^{G T}\right)$ and its parabolic limit $\left(\mathcal{M}_{f}^{p-s y s}\right)$ provide similar results (actually, they are superposed on Figure 2-right). Nevertheless the naive coupling provides a very different solution which is discontinuous at $x_{I+1 / 2}$.

Therefore, another strategy has to be developed in order to improve the behavior of the coupled numerical solution and recover the continuity of $v$ at $x_{I+1 / 2}$. To do so, we introduce some auxiliary unknowns $\mathbf{U}^{*}=$ $\left(u^{*}, v^{*}\right)^{T}$ on the left-hand side of the interface and solve a partial Riemann problem, see Figure 1. The RankineHugoniot condition through the 1-wave of speed $-\frac{a}{\varepsilon}$ yields

$$
u^{*}-u_{L}=a\left(v_{L}-v^{*}\right) .
$$

By requiring the continuity of the flux of $v$ through the interface $x_{I+1 / 2}$, we impose that

$$
\frac{u^{*}}{\varepsilon}=-\frac{a^{2}}{\sigma} \frac{v_{R}-v^{*}}{\frac{\Delta x}{2}}
$$




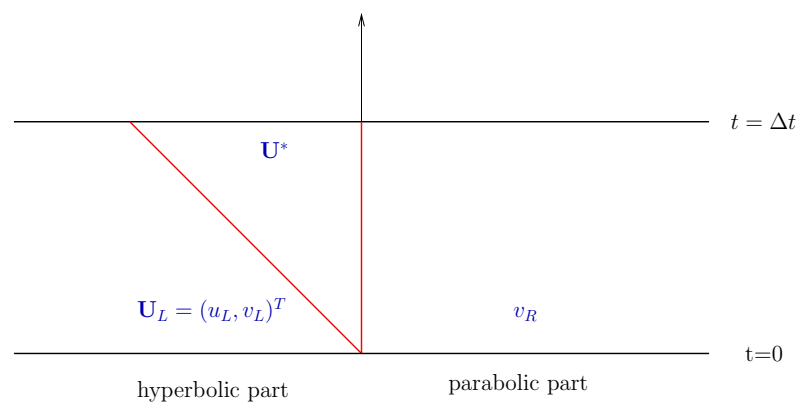

Figure 1. The interface states $\mathbf{U}^{*}=\left(u^{*}, v^{*}\right)$ are linked to $\left(u_{L}, v_{L}\right)$ by the 1-wave of speed $-a / \varepsilon$, and to $v_{R}$ by imposing the continuity of the numerical flux of $v$.

This condition is very natural since it ensure the conservation of $v$. Combining (50) and (51), we obtain the intermediate values

$$
\begin{aligned}
& v^{*}\left(W_{L}, v_{R}\right)=\left(\frac{1}{1+\frac{2 \varepsilon a}{\sigma \Delta x}}\right)\left(v_{L}+\frac{2 \varepsilon a}{\sigma \Delta x} v_{R}+\frac{u_{L}}{a}\right) . \\
& u^{*}\left(W_{L}, v_{R}\right)=\frac{1}{K_{\varepsilon}}\left(u_{L}+a\left(v_{L}-v_{R}\right)\right)
\end{aligned}
$$

It remains to plug these formulas in the numerical scheme (4) and (5) setting $i=I$ and replacing the numerical flux $\bar{u}\left(W_{I}^{n}, W_{I+1}^{n}\right) / K_{\varepsilon}$ and $\bar{v}^{-}\left(W_{I}^{n}, W_{I+1}^{n}\right)$ by $u^{*}\left(W_{I}^{n}, v_{I+1}^{n}\right)$ and $v^{*}\left(W_{I}^{n}, v_{I+1}^{n}\right)$. Then, the source term is once again treated in an implicit way in order to obtain an asymptotic preserving scheme which, after some computations, leads to

$$
\begin{aligned}
& v_{I}^{n+1}=v_{I}^{n}-\frac{\Delta t}{\varepsilon K_{\varepsilon} \Delta x}\left[u_{I}^{n}+a\left(v_{I+1}^{n}-v_{I}^{n}\right)-\bar{u}\left(W_{I-1}^{n}, W_{I}^{n}\right)\right], \\
& u_{I}^{n+1}=u_{I}^{n}-\frac{a^{2} \Delta t}{\varepsilon K_{\varepsilon} \Delta x}\left[v_{I+1}^{n}-\bar{v}\left(W_{I-1}^{n}, W_{I}^{n}\right)\right]-\frac{\sigma \Delta t}{\varepsilon^{2} K_{\varepsilon}} u_{I}^{n+1},
\end{aligned}
$$

while the parabolic flux is the cell $I+1$ is given by (34) and (53). Note that the numerical flux at the interface $x_{I+1 / 2}$ in (54) satisfies

$$
\lim _{\varepsilon \rightarrow 0} \frac{u^{*}}{\varepsilon}\left(\left(v_{I}^{n}, 0\right), v_{I+1}^{n}\right)=\frac{2 a^{2}}{\sigma} \frac{v_{L}-v_{R}}{\Delta x},
$$

which is not a consistent approximation for the flux of the expected heat equation that $v$ should satisfy. Nevertheless, the resulting coupling method provides very good results.

We illustrate this new coupling method in a simple test case. The 1D space domain is split in two parts: $(-1,0)$ and $(0,1)$. The model in the left part is the fine one $\left(\mathcal{M}_{f}^{G T}\right)$ and in the right part, the model is the coarse one $\left(\mathcal{M}_{c}^{G T}\right)$. We compare the numerical coupling method described above with the basic one, which amounts to set $u_{I+1}^{n}$ in the hyperbolic scheme (4)-(5) to define the numerical coupling flux. We also plot the results obtain with the full hyperbolic solution and the full parabolic solution. The initial condition is of Riemann type, with $v_{L}=1$ and $v_{R}=u_{L}=u_{R}=0$. The values of the parameters are set to: $\sigma=2$ and $a=1$. Each part is discretized with 30 cells. One can easily check that the basic coupling method introduces a jump at the coupling interface. Moreover, for $\varepsilon=0.1$ (right figure), though the full hyperbolic solution and the parabolic solution are superposed, the basic coupling still provides a jump at the coupling interface. Actually, this test case was the motivation of the construction of this new coupling method. We can check that in this latter case the results of the new coupling method are very good. If we go back to the case $\varepsilon=0.2$ (left figure), the hyperbolic solution 

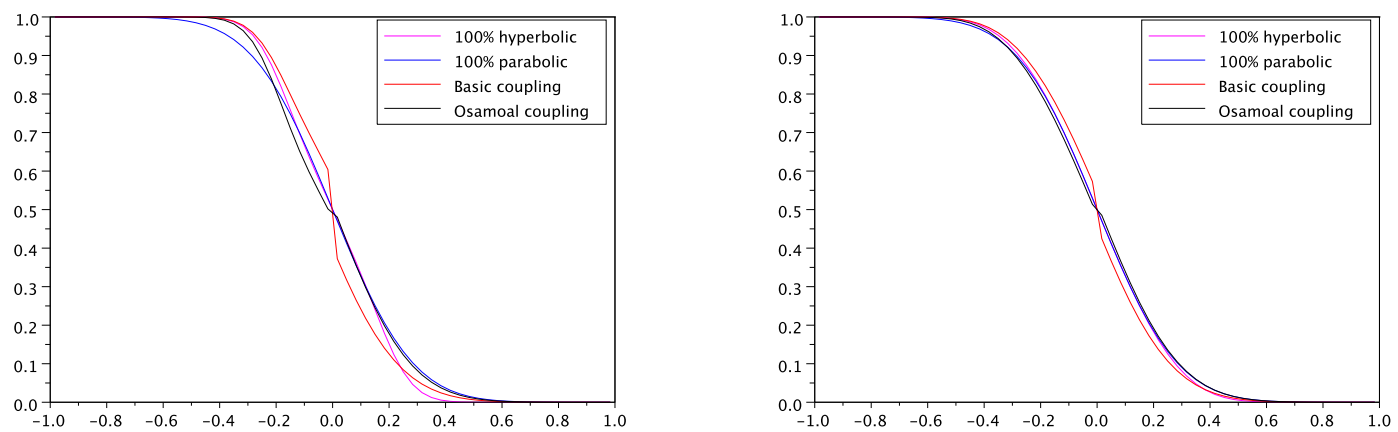

Figure 2. Approximate $v$ with $\varepsilon=0.2$ (left) and with $\varepsilon=0.1$ (right) at $t=0.05$.

and the parabolic solution are very different. Nonetheless, the new coupling method is close to the hyperbolic solution in the left part and close to the parabolic solution in the right part.

\subsection{The $p$-system}

We perform a similar coupling technique for the $p$-system. Here again the fine model $\left(\mathcal{M}_{f}^{p-s y s}\right)$ is solved on the left side of the interface and the coarse model $\left(\mathcal{M}_{c}^{p-s y s}\right)$ is solved on the right side of $x_{I}$. Because of the nonlinearity of both models, the design of an exact coupling strategy with a partial Riemann problem would lead to a nonlinear method. In order to avoid this, we make use of a relaxation method $[4,7]$ to linearize the $p$-system and we study the associated coupled partial Riemann problem in order to define the numerical fluxes. It takes the form

$$
\left\{\begin{array}{l}
\varepsilon \partial_{t} \tau-\partial_{x} u=0, \\
\varepsilon \partial_{t} u+\partial_{x} \pi(T, \tau)=\frac{-\sigma}{\varepsilon} u, \\
\partial_{t} T=\lambda(\tau-T) \\
(\tau, u, T)(x, 0)=\left(\tau_{L}, u_{L}, \tau_{L}\right)
\end{array} \quad \text { for }(x, t) \text { in } \mathcal{D}_{f}=\left(-\infty, x_{I}\right) \times[0, \infty)\right.
$$

where $\pi(\tau, T)=P(T)+a^{2}(T-\tau)$ denotes the relaxed pressure and we assume that the Whitham condition $a^{2}>\max _{s}\left|P^{\prime}(s)\right|$ is satisfied. The coarse model $\left(\mathcal{M}_{c}^{p-s y s}\right)$ to be solved on the right side of the interface reads

$$
\left\{\begin{array}{l}
\partial_{t} \tau+\frac{1}{\sigma} \partial_{x x} P(\tau)=0, \\
u=0, \\
\tau(x, 0)=\tau_{R},
\end{array} \quad \text { for }(x, t) \text { in } \mathcal{D}_{c}=\left(x_{I}, \infty\right) \times[0, \infty) .\right.
$$

Following the same strategy as the one used for the Goldstein-Taylor model, we introduce an interfacial state $\mathbf{U}^{*}=\left(\tau^{*}, u^{*}, \pi^{*}\right)^{T}$ on the left hand side of the interface. The left state and the interfacial state $\mathbf{U}^{*}$ are related by the Rankine-Hugoniot condition through the 1 -wave of speed $-\frac{a}{\varepsilon}$, i.e.

$$
\begin{aligned}
a\left(\tau^{*}-\tau_{L}\right) & =\left(u^{*}-u_{L}\right) \\
-a\left(u^{*}-u_{L}\right) & =\left(\pi^{*}-P\left(\tau_{L}\right)\right)
\end{aligned}
$$

since $\pi_{L}=P\left(\tau_{L}\right)$. First, we assume that the pressure is continuous through the interface, that is to say that there is only one pressure at the interface, namely $\pi^{*}$. In order to obtain a conservative method for $\tau$, the 
associated flux has to be the same through the interface $x_{I}$, which leads to the equality

$$
-\frac{1}{\varepsilon} u^{*}=\frac{1}{\sigma} \frac{P\left(\tau_{R}\right)-\pi^{*}}{\frac{\Delta x}{2}},
$$

which is nothing but the counterpart to (51). Combining equations (58), (59) and (60), we obtain

$$
\begin{aligned}
\tau^{*} & =\tau_{L}+\frac{1}{a}\left(u^{*}-u_{L}\right), \\
u^{*} & =\left(\frac{1}{1+\frac{\sigma \Delta x}{2 \varepsilon a}}\right)\left(u_{L}-\frac{1}{a}\left(P\left(\tau_{R}\right)-P\left(\tau_{L}\right)\right)\right), \\
\pi^{*} & =\left(\frac{1}{1+\frac{2 \varepsilon a}{\sigma \Delta x}}\right)\left(P\left(\tau_{L}\right)+\frac{2 a \varepsilon}{\sigma \Delta x} P\left(\tau_{R}\right)+a u_{L}\right) .
\end{aligned}
$$

As in the case of the Goldstein-Taylor model, the values $u^{*}$ and $\pi^{*}$ are plugged in the well-balanced scheme (21) for the cell $i=I$. Following the same computations which leads to the scheme (25)-(26) and after the implicit modification of the source term, one obtains

$$
\begin{aligned}
& \tau_{I}^{n+1}=\tau_{I}^{n}-\frac{\Delta t}{\varepsilon L_{\varepsilon} \Delta x}\left[u_{I}^{n}+a\left(P\left(\tau_{I+1}^{n}\right)-P\left(\tau_{I}^{n}\right)\right)-u_{I-1 / 2}^{n}\right], \\
& u_{I}^{n+1}=u_{I}^{n}-\frac{\Delta t}{\varepsilon L_{\varepsilon} \Delta x}\left[P\left(\tau_{I+1}^{n}\right)-\Pi\left(W_{I-1}^{n}, W_{I}^{n}\right)\right]-\frac{\sigma \Delta t}{\varepsilon^{2} L_{\varepsilon}} u_{I}^{n+1},
\end{aligned}
$$

where $u_{I-1 / 2}^{n}$ is given by $(22)$ and

$$
\Pi\left(W_{L}, W_{R}\right)=\frac{1}{2}\left(P\left(\tau_{L}\right)+P\left(\tau_{R}\right)\right)-\frac{a}{2}\left(u_{R}-u_{L}\right) .
$$

Remark 3.1. Other coupling conditions could have been chosen, for instance the continuity of the specific volume $\tau$ through the interface instead of the continuity of the pressure. However this condition requires to solve a nonlinear problem in order to determine the interfacial state.

Let us now present the numerical results of two test cases. The pressure law is $P(\tau)=\tau^{-2}$ and $\sigma=2$. The initial data corresponds to a Riemann problem with $\tau_{L}=2, \tau_{R}=1$ and $u_{L}=u_{R}=0$. Once again, each part of the computational domain is discretized in 30 cells. Two tests with different values of $\varepsilon$ are provided in Figure 3. In the right figure, $\varepsilon$ is sufficiently small to obtain very close solutions for the fully hyperbolic case and for the fully parabolic case. The solution of our coupling method is also very close to these two solutions, as expected. In the contrary, the basic coupling method, which amounts to use the hyperbolic flux with $u_{I+1}=0$, introduces a jump at the coupling interface. In the left figure, the solutions of the fully hyperbolic case and of the fully parabolic case are different since $\varepsilon$ is greater than in the previous case. A jump of $\tau$ appears with the basic coupling method which is larger than in the case of a smaller $\varepsilon$. Indeed, some complementary numerical experiments let us think that the amplitude of this jump to tend to 0 when $\varepsilon$ goes to 0 . The result provided by our coupling method is very satisfying. It follows the shape of the hyperbolic solution in the left part and the shape of the parabolic solution in the right part. As in the Goldstein-Taylor case, the transmission through the coupling interface with our new coupling method is however not fully perfect. Indeed, a small jump can be detected for both values of $\varepsilon$ which can be probably related to the lack of consistency of the coupling flux when $\varepsilon$ tends to 0 .

\section{Conclusion}

During this project, we have proposed asymptotic preserving schemes for the parabolic limit of the GoldsteinTaylor system and of the $p$-system. The core of these schemes and their analysis was already present in $[14,15]$ 

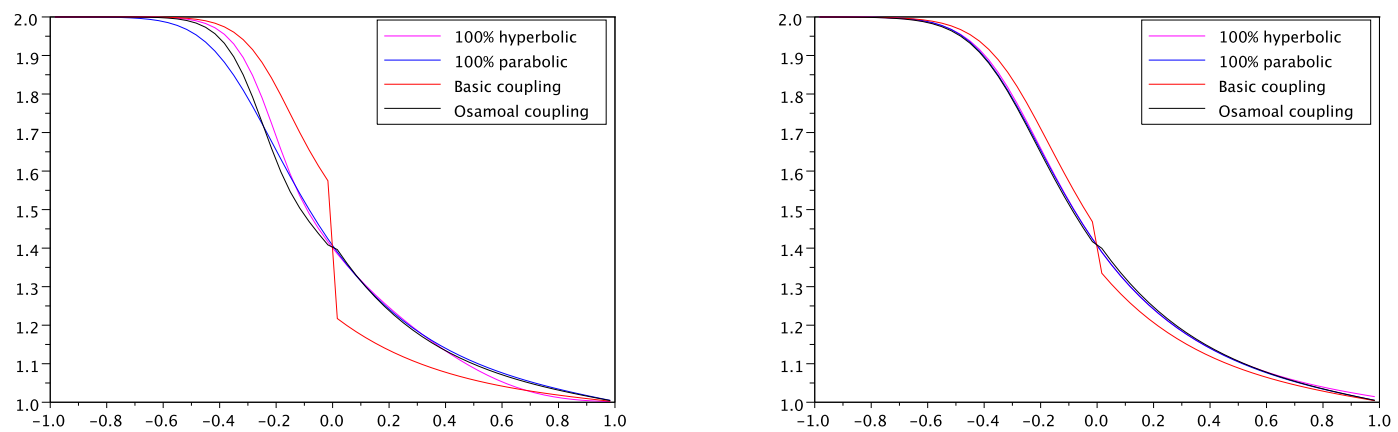

FiguRE 3. Approximate $\tau$ with $\varepsilon=0.4$ (left) and with $\varepsilon=0.1$ (right) at $t=0.2$.

as far as the Goldstein-Taylor model is concerned. We also have provided an original coupling method between a hyperbolic system (with source term) and its parabolic limit in order to remove the jumps that the classical coupling method makes appear. The remaining work about the model adaptation concerns the dynamical part: definition of error indicators and development of the fully dynamic adaptive algorithm, following [5,21]. This is under investigation, as well as the extension to more complex models.

\section{REFERENCES}

[1] A. Ambroso, C. Chalons, F. Coquel, T. Galié, E. Godlewski, P.-A. Raviart, and N. Seguin. The drift-flux asymptotic limit of barotropic two-phase two-pressure models. Commun. Math. Sci., 6(2):521-529, 2008.

[2] M. R. Baer and J. W. Nunziato. A two-phase mixture theory for the deflagration-to-detonation transition (DDT) in reactive granular materials. Int. J. Multiphase Flow, 12:861-889, 1986.

[3] C. Berthon and R. Turpault. Asymptotic preserving HLL schemes. Numer. Meth. P.D.E., 27(6):1396-1422, 2010.

[4] F. Bouchut. Nonlinear stability of finite volume methods for hyperbolic conservation laws and well-balanced schemes for sources. Frontiers in Mathematics. Birkhäuser Verlag, Basel, 2004.

[5] C. Cancès, F. Coquel, E. Godlewski, H. Mathis, and N. Seguin. Model adaptation for hyperbolic systems with relaxation. In preparation, 2012.

[6] C. Chalons, F. Coquel, E. Godlewski, P.-A. Raviart, and N. Seguin. Godunov-type schemes for hyperbolic systems with parameter-dependent source. The case of Euler system with friction. Math. Models Methods Appl. Sci., 20(11):2109-2166, 2010.

[7] F. Coquel, E. Godlewski, B. Perthame, A. In, and P. Rascle. Some new Godunov and relaxation methods for two-phase flow problems. In Godunov methods (Oxford, 1999), pages 179-188. Kluwer/Plenum, New York, 2001.

[8] R. Eymard, T. Gallouët, and R. Herbin. Finite volume methods. In Handbook of numerical analysis, Vol. VII, Handb. Numer. Anal., VII, pages 713-1020. North-Holland, Amsterdam, 2000.

[9] E. Franck, C. Buet, and B. Després. Asymptotic preserving finite volumes discretization for non-linear moment model on unstructured meshes. In Finite volumes for complex applications, VI (Prague, 2011), pages 467-474. Springer, 2011.

[10] T. Gallouët and J.-C. Latché. Compactness of discrete approximate solutions to parabolic PDEs-Application to a turbulence model. To appear in Communications on Pure and Applied Analysis.

[11] E. Godlewski, K.-C. Le Thanh, and P.-A. Raviart. The numerical interface coupling of nonlinear hyperbolic systems of conservation laws. II. The case of systems. M2AN Math. Model. Numer. Anal., 39(4):649-692, 2005.

[12] E. Godlewski and P.-A. Raviart. The numerical interface coupling of nonlinear hyperbolic systems of conservation laws. I. The scalar case. Numer. Math., 97(1):81-130, 2004.

[13] L. Gosse and A.-Y. LeRoux. Un schéma-équilibre adapté aux lois de conservation scalaires non-homogènes. C. R. Acad. Sci. Paris Sér. I Math., 323(5):543-546, 1996.

[14] L. Gosse and G. Toscani. An asymptotic-preserving well-balanced scheme for the hyperbolic heat equations. C. R. Math. Acad. Sci. Paris, 334(4):337-342, 2002.

[15] L. Gosse and G. Toscani. Space localization and well-balanced schemes for discrete kinetic models in diffusive regimes. SIAM J. Numer. Anal., 41(2):641-658 (electronic), 2003. 
[16] J. M. Greenberg and A.-Y. LeRoux. A well-balanced scheme for the numerical processing of source terms in hyperbolic equations. SIAM J. Numer. Anal., 33(1):1-16, 1996.

[17] A. Harten, P. D. Lax, and B. van Leer. On upstream differencing and Godunov-type schemes for hyperbolic conservation laws. SIAM Rev., 25(1):35-61, 1983.

[18] T. Hibiki and M. Ishii. One-dimensional drift-flux model and constitutive equations for relative motion between phases in various two-phase flow regimes. International Journal of Heat and Mass Transfer, 46:4935-4948, 2003.

[19] S. Jin. Efficient asymptotic-preserving (AP) schemes for some multiscale kinetic equations. SIAM J. Sci. Comput., 21(2):441454 (electronic), 1999.

[20] S. Jin, L. Pareschi, and G. Toscani. Diffusive relaxation schemes for multiscale discrete-velocity kinetic equations. SIAM J. Numer. Anal., 35(6):2405-2439 (electronic), 1998.

[21] H. Mathis and N. Seguin. Model adaptation for hyperbolic systems with relaxation. In Finite volumes for complex applications, VI (Prague, 2011), pages 673-681. Springer, 2011. 